\title{
ON RINGS WHOSE INJECTIVE INDECOMPOSABLE MODULES ARE FINITELY GENERATED
}

\author{
Guenter KraUse
}

(Received June 20, 1969)

0. Introduction. It is well known that a commutative noetherian ring with identity is artinian if and only if its injective (directly) indecomposable modules are finitely generated. If $R$ is left noetherian, but not necessarily commutative, the latter property implies the minimum condition on left ideals (cf. [4, Corollary 2.3]), the converse, however, is false in general (cf. [12,p. 375]). Therefore, two interesting questions arise:

1. What additional ring theoretical properties must a left artinian ring have to make its injective indecomposable left modules finitely generated.

2. What exactly is the class of all rings with identity for which the minimum condition on left ideals is equivalent to the maximum condition on left ideals plus the property that injective indecomposable left modules are finitely generated.

Similar problems have been investigated by numerous authors, the best contribution to 1 can be found in [12]. The purpose of this paper is to give a few partial solutions of these two problems. In $\S 2$, we restrict ourselves to reduced left artinian rings, i. e. rings which are direct sums of division rings modulo their Jacobson radical $J$. If $R$ is such a ring and if $E_{R}(R / J)$ denotes the injective hull of the left $R$-module $R / J$, it turns out that injective indecomposable left $R$-modules are finitely generated if and only if the module $E_{R}(R / J) / R / J$ has finite dimension in the sense of Goldie (Theorem 2.2). In $\$ 3$ we restrict the discussion further to rings which are direct sums of finitely many local left artinian rings. These rings have the property that their injective indecomposable modules are cyclic and their submodules are linearly ordered if and only if the dimension of $E_{R}(R / J) / R / J$ is equal to the number of prime ideals of $R$ which are large left ideals. In $\$ 4$, we show that for the class of all rings which are commutative modulo the square of their Jacobson radical the minimum condition on left ideals is equivalent to the maximum condition on left ideals plus the property that injective indecomposable left modules are finitely generated (Theorem 4.1).

Definitions And Notations. Throughout this paper each ring $R$ will 
be a (not necessarily commutative) ring with identity element 1 , each module $M$ will be a unitary left $R$-module in the sense that $1 m=m$ for all $m \in M$. $E(M)=E_{R}(M)$ denotes the injective hull of the left $R$-module $M, \pi(M)$ is a set of representatives of the isomorphism classes of indecomposable injective submodules of $E(M)$.

A submodule $N$ of $M$ is large in $M$ (denoted by $N \subseteq^{\prime} M$ ), and $M$ is an essential extension of $N$ if $N \cap X \neq 0$ for every nonzero submodule $X$ of $M$. Dually, a submodule $N$ of $M$ is small in $M$, if $X+N \neq M$ for all proper submodules $X$ of $M$. A module $M$ is said to be small, if it is a small submodule of some module. $X_{l}=X_{l}(R)=\{r \in R \mid r X=0\}$ denotes the left annihilator in $R$ of the subset $X$ of the left $R$-module $M . Y_{r}=Y_{r}(M)=\{m \in M \mid Y m=0\}$ denotes the right annihilator in $M$ of the subset $Y$ of $R$. A prime ideal $P$ of $R$ is called associated with the module $M$, if $P=N_{\imath}^{\prime}$ for all nonzero submodules $N^{\prime}$ of some submodule $N$ of $M$. The set $\operatorname{ch}(M)$ of all prime ideals associated with $M$ is called the characteristic of $M$. The set $\operatorname{supp}(M)$ is the set of all prime ideals of $R$ which contain the left annihilator $M_{l}$ of $M$.

$$
\begin{aligned}
J=J(R) & =\text { Jacobson radical of the } \operatorname{ring} R . \\
S(M) & =\text { socle of the module } M . \\
C(M) & =\text { heart of } M=M \cap \bigcap_{\beta \in J\left(\operatorname{Hom}_{R}(E(M), E(M))\right)} \operatorname{ker} \beta \\
Z(M) & =\text { singular submodule of } M . \\
M \oplus N & =\text { direct sum of the modules } M \text { and } N . \\
\bigoplus_{i \in I} M_{i} & =\text { (discrete) direct sum of the modules } M_{i} . \\
\operatorname{dim}_{R}(M) & =\operatorname{dim}(M)=\text { Goldie-dimension of the module } M .
\end{aligned}
$$

A ring $R$ is called local, if $R / J$ is a division ring, $R$ is called reduced, if $R / J$ is a direct sum of division rings.

\section{Preliminaries.}

1. 1. Definition. (Bass[2]) Let $\Sigma$ be a non-empty family of cyclic left $\mathrm{R}$-modules. A $\Sigma$-module is a nonzero direct sum of modules eachof which is isomorphic to a member of $\Sigma$. $\Sigma$ is called an injective basis for $R$ if every nonzero injective $R$-module contains a $\Sigma$-module.

1. 2. EXAMPLES. (a) $\Sigma=\{R / I \mid I$ any proper left ideal $\}$ is an injective basis for any ring $R$ with identity (b) (Matlis[11]) if $R$ is a commutative noetherian ring, then $\Sigma=\{R / P \mid P$ any prime ideal $\}$ is an injective basis for $R$.

1. 3. Definition. A left noetherian ring $R$ is called a Matlis ring if the 
set $\Sigma=\{R / P \mid P$ any prime ideal $\}$ is an injective basis for $R$.

1. 4. REMARK. Matlis rings (although not under this name) have been studied in the author's previous papers [6] and [7]. Since a left noetherian left uniform prime ring has no zero divisors by [5, Lemma 3.3 and Lemma 3.8], it is clear that the class of all Matlis rings is a subclass of the class of all left noetherian rings whose prime ideals are completely prime. The two classes, however, are not the same (cf [10, Exemple 10.1]). In certain cases one knows the inner structure of Matlis rings quite well, one result being the following:

1. 5. THEOREM. A ring $R$ with minimum condition on left ideals is a Matlis ring if and only if it is reduced.

PROOF. cf. [7, Theorem 3.6]

1.6. COROllary. A ring is a semi-simple Matlis ring with minimum condition if and only if it is a direct sum of finitely many division rings.

The following result will be needed in section 3 .

1. 7. LemmA. Let $R$ be a left noetherian ring, let $\Sigma=\left\{E_{i}, i \in I\right\}$ be a set of injective left $R$-modules with small socles, and let $F$ be a direct sum of modules isomorphic to elements in $\Sigma$. Then $S(F)$ is small in $F$.

ProOF. Without loss of generality it may be assumed that $\Sigma$ consists of injective indecomposable modules. For let $E$ be injective with small socle and let $E$ be the direct sum of injective indecomposable modules $E_{k}, k \in K$. If $S\left(E_{\kappa_{0}}\right)+X=E_{k_{0}}$ with $X \neq E_{k_{0}}$ for some $K_{0} \in K$, then $S(E)+\left(X \oplus \bigoplus_{k \neq k_{0}} E_{k}\right)=E$, where $X \oplus \bigoplus_{k \neq k_{0}} E_{k} \neq E$, a contradiction to the smallness of $S(E)$ in $E$.

Assume now that $S(F)$ is not small in $F$, let $N \neq F$ be a submodule of $F$ such that $S(F)+N=F$. Since $F$ is injective, $E(N)$ can be imbedded in $F$, whence $F=S(F)+E(N)$. Since $S(F) \nsubseteq N, N$ cannot be a large submodule of $F$, so that $E(N) \neq F$. Thus there exists a nonzero submodule $M$ such that $F=M \oplus E(N)$, whence $S(F)=S(M) \oplus S(E(N))$. Therefore, $F=S(F)+E(N)$ $=(S(M) \oplus S(E(N)))+E(N)=S(M) \oplus E(N)$. Therefore, $S(M)$ is injective and hence a direct sum of modules in $\Sigma$ by [11, Theorem 2.5] and[1, Theorem 1]. But this implies that some module in $\Sigma$ is equal to its socle, contradicting the assumption that all elements in $\Sigma$ have small socle. Therefore $S(F)$ must be small in $F$. 


\section{Reduced left artinian rings.}

2. 1. Proposition. Let $R$ be a left artinian ring and let $P_{1}, \cdots, P_{n}$ denote the finitely many prime ideals of $R$. Then:

(a) If $R$ is reduced, then $R / J \cong_{R} R / P_{1} \oplus \cdots \oplus R / P_{n}$ and $E_{R}(R / J)$ $\cong_{R} E_{R}\left(R / P_{1}\right) \oplus \cdots \oplus E_{R}\left(E / P_{n}\right)$.

(b) If $R$ is a direct sum of local rings, then $\operatorname{Hom}_{R}\left(E_{R}(R / P), E_{R}(R / P)\right)$ is a left and right perfect local ring with nilpotent Jacobson radical for every prime ideal $P$. Furthermore, the ring $\operatorname{Hom}_{R}\left(E_{R}(R / J), E_{R}(R / J)\right)$ is isomorphic to the direct sum of the rings $\operatorname{Hom}_{R}\left(E_{R}\left(R / P_{i}\right), E_{R}\left(R / P_{i}\right)\right)$, $i=1, \cdots, n$.

PROOF. (a) It follows from [7, Theorem 3.6] that prime ideals of $R$ are maximal left ideals. Since $J=\bigcap_{i=1}^{n} P_{i}, R / J$ is a left $R$-module of length $n$. Since $R / J$ can be imbedded monomorphically in the module $\bigoplus_{i=1}^{n} R / P_{i}$ of length $n$, it follows that this imbedding is an isomorphism from ${ }_{n} R / J$ onto $\bigoplus_{i=1}^{n} R / P_{i}$ and can be extended to an isomorphism of $E_{R}(R / J)$ onto $\bigoplus_{i=1}^{n} E_{R}\left(R / P_{i}\right)$.

(b) Since prime ideals are maximal left ideals by [6, Satz 4.2], each $E_{R}\left(R / P_{i}\right)$ is injective and indecomposable, which by [9, Théorème 2.1] implies that $H_{i}=\operatorname{Hom}_{R}\left(E_{R}\left(R / P_{i}\right), E_{R}\left(R / P_{i}\right)\right)$ is a local ring. Thus it suffices to show that the Jacobson radical of each $H_{i}$ is nilpotent. Let $P$ be a prime ideal, let $E=E_{R}(R / P)$. Since $R$ is left artinian, $P^{n}=P^{n+1}$ for some $n$. Since $\operatorname{supp}(M)$ $=\operatorname{ch}(M)$ for every left $R$-module $M$ by [6, Satz 4.2], we get

$$
\operatorname{ch}\left(E / P_{r}^{n}(E)\right)=\operatorname{supp}\left(E / P_{r}^{n}(E)\right) \subseteq \operatorname{supp}(E)=\operatorname{ch}(E)=\{P\}
$$

Therefore, $E / P_{r}^{n}(E)=0$, since otherwise $E / P_{r}^{n}(E)$ would contain a nonzero submodule whose annihilator is $P$, contrary to the fact that $P^{n}=P^{n+1}$. Thus $P^{n} E=0$. Let now $\boldsymbol{\varphi}_{1}, \boldsymbol{\phi}_{2}, \cdots, \boldsymbol{\varphi}_{n} \in g=J\left(\operatorname{Hom}_{R}(E, E)\right)$. By [6, Satz 4.2 and Lemma 3.5]

$$
P_{r}(E) \subseteq C(E)=g_{l}(E) \subseteq\left(\phi_{1}\right)_{l}(E)
$$

whence $\left(P^{n-1} e\right) \boldsymbol{\varphi}_{1}=0$ for every $e \in E$. Thus

$$
\left(P^{n-2} \boldsymbol{e}\right) \boldsymbol{\phi}_{1} \subseteq P_{r}(E) \subseteq\left(\phi_{2}\right)_{l}(E)
$$

for every $e \in E$, whence $\left(P^{n-2} e\right) \phi_{1} \phi_{2}=0$. Continuing this way, we finally obtain $(P e) \varphi_{1} \phi_{2} \cdots \varphi_{n-1}=0$ and hence $e \varphi_{1} \cdots \varphi_{n-1} \in P_{r}(E) \subseteq\left(\phi_{n}\right)_{l}(E)$ for every $e \in E$. This shows that $g^{n}=0$. 
To prove the last statement, let $\phi \in \operatorname{Hom}_{R}(E(R / J), E(R / J))$. By (a), $E_{R}(R / J)=E_{1} \oplus \cdots \oplus E_{n}$, where $E_{i} \cong E_{R}\left(R / P_{i}\right), i=1, \cdots, n$. Assume $E_{i} \varphi \nsubseteq E_{i}$ for some fixed $i$, Then $x \boldsymbol{\varphi}=x_{1}+\cdots+x_{n}$, where $x_{j} \in E_{j}$ and $x_{j} \neq 0$ for at least one $j \neq i$. Since maximal left ideals of $R$ are prime ideals (cf. [7, Theorem 3.6] and since $\operatorname{supp}\left(E_{j}\right)=\operatorname{ch}\left(E_{j}\right)=\left\{P_{j}\right\}$ by [6, Satz 4.2], it follows that $\left(x_{j}\right)_{l} \subseteq P_{j}$. Thus

$$
P_{j} \supseteq\left(x_{j}\right)_{l} \supseteq \bigcap_{k=1}^{n}\left(x_{k}\right)_{l}=(x \boldsymbol{\varphi})_{l} \supseteq x_{l} \supseteq\left(E_{i}\right)_{l}
$$

whence $P_{j} \in \operatorname{supp}\left(E_{i}\right)=\operatorname{ch}\left(E_{i}\right)=\left\{P_{i}\right\}$, a contradiction. Hence $E_{i} \boldsymbol{\Phi} \subseteq E_{i}$ for all $i=1, \cdots, n$. If we define $\phi \lambda=\left(\phi\left|E_{1}, \cdots, \phi\right| E_{n}\right)$ for all $\phi \in \operatorname{Hom}_{R}\left(E_{R}(R / J)\right.$, $\left.E_{R}(R / J)\right)$, then $\lambda$ is clearly a ring isomorphism of the $\operatorname{ring} \operatorname{Hom}_{R}\left(E_{R}(R / J)\right.$, $\left.E_{R}(R / J)\right)$ onto the direct sum of the rings $\operatorname{Hom}_{R}\left(E_{i}, E_{i}\right), i=1, \cdots, n$.

2. 2. THEOREM. The following properties of a ring $R$ are equivalent:

(1) $\{$ (a) $R$ is a Matlis ring.

(b) Injective indecomposable modules are finitely generated.

$$
\left\{\begin{array}{l}
\text { (a) } R \text { is a reduced left artinian ring. } \\
\text { (b) } \operatorname{dim}_{R}\left[E_{R}(R / J) / R / J\right]<\infty
\end{array}\right.
$$

Proof. (1) $\rightarrow$ (2) Since $R$ is left artinian by [4, Corollary 2.3], (2a) follows from [6, Hilfssatz 4.1] and [7, Theorem 3.6]. Since $R / J$ is a noetherian left $R$-module, $E(R / J)$ is a direct sum of finitely many indecomposable modules. Therefore, $E(R / J)$ is finitely generated and hence noetherian, since $R$ is left noetherian. Thus $E(R / J) / R / J$ is noetherian which implies in particular $(2 \mathrm{~b})$. $(2) \rightarrow(1)$ : Let $P_{1}, \cdots, P_{n}$ denote the finitely many prime ideals of $R$. Then $R / J \cong_{R} \bigoplus_{i=1}^{n} R / P_{i}$ and $E_{R}(R / J) \cong \bigoplus_{i=1}^{n} E\left(R / P_{i}\right)$ by Proposition 2.1. By [7, Theorem 3.6] $R$ is a Matlis ring, so (1a) holds. Let now $E=E_{R}(R / P)$ be an injective indecomposable module and assume without loss of generality that $P=P_{1}$. Then

$$
\begin{aligned}
E_{R}(R / P) / R / P & \cong\left(E\left(R / P_{1}\right) \oplus \bigoplus_{i=2}^{n} R / P_{i}\right) / \bigoplus_{i=1}^{n} R / P_{i} \subseteq \bigoplus_{i=1}^{n} E\left(R / P_{i}\right) / \bigoplus_{i=1}^{n} R / P_{i} \\
& \cong E(R / J) / R / J,
\end{aligned}
$$

which implies that $E(R / P) / R / P$ has finite dimension. Since $P$ is a maximal left ideal, $R / P$ is the socle of $E=E(R / P)$. Define $S_{1}(E)=S(E)$ and $S_{i}(E) / S_{i-1}(E)$ $=S\left(E / S_{i-1}(E)\right)$ for $i>1$. Since $R$ is left artinian, $S(M)=J_{r}(M)$ for every unitary 
left $R$-module $M$. Therefore, the nilpotence of $J$ shows that the lower Loewyseries $S_{1}(E) \subseteq S_{2}(E) \subseteq \cdots$ terminates at $E$ after finitely many steps. Thus it suffices to show that for every $i$ the module $E / S_{i}(E)$ has finite Goldie-dimension since this implies that $S_{i+1}(E) / S_{i}(E)=S\left(E / S_{i}(E)\right.$ ) is a direct sum of finitely many simple modules and thus finitely generated. From this (1b) follows immediately since $S_{1}(E)=R / P$ is simple and extensions of finitely generated modules by finitely generated modules are finitely generated.

By the above $E / S_{1}(E)$ has finite dimension. Assume that $E / S_{i}(E)$ has finite dimension $m$ for $i \geqq 1$. Then $S\left(E / S_{i}(E)\right)=S_{i+1}(E) / S_{i}(E)$ is a direct sum of $m$ simple modules and thus of the form $\bigoplus_{i=1}^{m} R / P_{i}$ with prime ideals $P_{i}$. Since $R$ is left artinian, every module has a large socle, so that $E\left(E / S_{i}(E)\right) \cong \bigoplus_{i=1}^{m} E\left(R / P_{i}\right)$. Thus,

$$
\begin{aligned}
E / S_{i+1}(E) & \cong E / S_{i}(E) / S_{i+1}(E) / S_{i}(E) \subseteq E\left(E / S_{i}(E)\right) / S_{i+1}(E) / S_{i}(E) \\
& \cong \bigoplus_{i=1}^{m} E\left(R / P_{i}\right) / \bigoplus_{i=1}^{m} R / P_{i} \cong \bigoplus_{i=1}^{m}\left[E\left(R / P_{i}\right) / R / P_{i}\right],
\end{aligned}
$$

where the last isomorphism is given by

$$
\boldsymbol{\varphi}:\left(e_{1}+\cdots+e_{m}\right)+\bigoplus_{i=1}^{m} R / P_{i} \rightarrow\left(e_{1}+R / P_{1}\right)+\cdots+\left(e_{m}+R / P_{m}\right) .
$$

Since each $E\left(R / P_{i}\right) / R / P_{i}$ has finite dimension, $E / S_{i+1}(E)$ can be embedded in a direct sum of finitely many finite dimensional modules and is thus finite dimensional.

Condition (2b) in the theorem is indispensible, since there exists even a local left artinian ring which has no finitely generated injective modules.

2. 3. EXAMPLE (Rosenberg and Zelinsky [12, p. 375]). Let $K$ be a field, $\sigma$ an isomorphism of $K$ into itself such that $[K: K \sigma]=\infty$. Define a $K$-bimodule $N$ in the following way: As left $K$-module, $N$ is isomrphic to $K$, on the right define $n k=(k \sigma) n$ for $k \in K, n \in N$. If we define $N^{2}=0$, the two-sided $K$-module direct sum $R=K \oplus N$ is a ring with minimum condition on left ideals with Jacobson radical $N . R / N$ is a field, whence $R$ is a local ring. But $R$ admits no injective finitely generated modules by $[12$, p. 375].

2. 4. REMARK. Together with Theorem 2.1 the preceding example shows that the module $E_{R}(R / N)$ is a module with Goldie-dimension 1 with an infinite dimensional factor module $E_{R}(R / N) / R / N$. 


\section{Finite direct sums of local left artinian rings.}

3.1. Notation. If $M$ is a left $R$-module and $A$ is a subset of $\operatorname{Hom}_{R}(M, M)$, and $N$ a subset of $M$, we define $A_{l}=\{m \in M \mid m A=0\}$ and $N_{r}=$ $\left\{\beta \in \operatorname{Hom}_{\mathrm{R}}(M, M) \mid N \beta=0\right\}$. $A_{l r}$ is called the closure of $A$.

3.2. THEOREM. The following properties of the ring $R$ are equivalent:
(a) $R$ is a Matlis ring.
(b) For every prime ideal $P$ of $R$ there exists an integer (c) $n=n(P)>0$ such that $P^{n} E_{R}(R / P)=0$.
(c) Injective indecomposable left $R$-modules are finitely generated.

(2) $\left\{\begin{array}{l}\text { (a) } R \text { is a Matlis ring. } \\ \text { (b) } R \text { has the Artin-Rees-property for left ideals. } \\ \text { (c) Injective indecomposable left } R \text {-modules are finitely generated. }\end{array}\right.$

(3) $\left\{\begin{array}{l}\text { (a) } R \text { is a direct sum of finitely many local left artinian rings. } \\ \text { (b) The Jacobson radical of } \operatorname{Hom}_{R}\left(E_{R}(R / J), E_{R}(R / J)\right) \text { is the closure } \\ \text { of a finitely generated right ideal. }\end{array}\right.$

(4) $\left\{\begin{array}{l}\text { (a) } R \text { is a direct sum of finitely many local left artinian rings. } \\ \text { (b) } \operatorname{dim}_{R}[E(R / J) / R / J]<\infty \text {. }\end{array}\right.$

Proof. (1) $\rightarrow(2):$ By [6, Satz 4.2] it suffices to show that $\operatorname{ch}(M)=\operatorname{supp}(M)$ for every left $R$-module $M$. Let $E$ be injective and indecomposable, $E \cong E_{R}(R / P)$ for some prime ideal $P$, and let $Q \in \operatorname{supp}(E)$. Since $P^{n} \subseteq Q$ for some natural number $n, P=Q$ since prime ideals of $R$ are maximal. Thus $\operatorname{ch}(E)=\operatorname{supp}(E)$. If $M$ is any module, $E(M)=\bigoplus_{i \in I} E_{i}$ with injective indecomposable modules $E_{i}$ (see [11, Theorem 2.5]). Since $R$ is left artinian, there exist finitely many $E_{k}, k=1, \cdots, n$ among the $E_{i}, i \in I$ such that $E(M)_{l}=\bigcap_{k=1}^{n}\left(E_{k}\right)_{l}$. Thus, if $Q \in \operatorname{supp}(M), Q$ contains at least one of the $\left(E_{k}\right)_{l}, k=1, \cdots, n$, whence $Q \in \operatorname{supp}\left(E_{k}\right)=\operatorname{ch}\left(E_{k}\right) \subseteq \operatorname{ch}(E(M))=\operatorname{ch}(M)$.

$(2) \rightarrow$ (3): (3a) follows from [6, Satz 4.2]. By 2.1 and (2a), $E=E_{R}(R / J)$ is finitely generated and thus artinian. Therefore, by [3, Theorem 3.1], $C(E)=\bigcap_{i=1}^{n}$ $\operatorname{ker} \beta_{i}$ for finitely many $\beta_{i}$ in the Jacobson radical $g$ of $H=\operatorname{Hom}_{R}\left(E_{R}(R / J)\right.$, $\left.E_{R}(R / J)\right)$. Since $\operatorname{ker} \beta_{i}=\left(\beta_{i}\right)_{l}(E)=\left(\beta_{i} H\right)_{l}(E)$ and since $C(E)=g_{l}(E)$, we get 


$$
g_{l}(E)=\bigcap_{i=1}^{n}\left(\beta_{i} H\right)_{l}=\left(\sum_{i=1}^{n} \beta_{i} H\right)_{l}
$$

whence $g_{l r}=\left(\sum_{i=1}^{n} \beta_{i} H\right)_{l r}$. If $\beta \in g_{l r}$, then $C(E) \beta=g_{l} \beta=0$, whence $C(E) \subseteq \operatorname{ker} \beta$.

Since $C(E)$ is a large submodule of $E, \operatorname{ker} \beta \subseteq{ }^{\prime} E$, whence $\beta \in g$ by [3, Theorem 3. 1]. Thus $g=g_{l r}$ and (3b) is proved.

$$
\begin{aligned}
& (3) \rightarrow(4): \text { If } g=\left(\sum_{i=1}^{n} \beta_{i} H\right)_{l r} \text {, then } C(E(R / J))=g_{l}=\left(\sum_{i=1}^{n} \beta_{i} H\right)_{l r l}=\left(\sum_{i=1}^{n} \beta_{i} H\right)_{l} \\
= & \bigcap_{i=1}^{n}\left(\beta_{i} H\right)_{l}=\bigcap_{i=1}^{n} \operatorname{ker} \beta_{i} \text {. Since } E(R / J) / \operatorname{ker} \beta_{i} \cong E(R / J) \beta_{i} \subseteq E(R / J) \text { for every }
\end{aligned}
$$
$i$ and since $E(R / J)$ is finite demensional, this implies that $E(R / J) / C(E(R / J))$ is finite demensional. Since $R / J$ is a semi-simple module and since $C(E(R / J))$ $=S(E(R / J))$ by $[7$, Theorem 4.2$]$, this implies (4b).

$(4) \rightarrow(1):$ It follows from [6, Satz 4.2] that $R$ satisfies (1a) and that $\operatorname{ch}(M)=\operatorname{supp}(M)$ for every left $R$-module $M$. Since $\operatorname{ch}(E(R / P))=\{P\}$ for every prime ideal $P$, it follows that $P / E(R / P)_{l}$ is the lower Baer nil radical of the ring $R / E(R / P)_{l}$ and is thus nilpotent since $R$ is left noetherian. But this implies (1b), and (1c) follows from Theorem 2.2.

3. 3. REMARK. It would be interesting to know whether in the situation of the preceding theorem the Jacobson radical of $\operatorname{Hom}_{R}(E(R / J), E(R / J))$ is itself a finitely generated right ideal. In any case, condition (3b) of the theorem is indispensible as Example 2.3 shows. It may be noted that with the notation of this example it follows from 2.1 that the endomorphism ring of the module $E_{R}(R / N)$ is a left and right perfect local ring with nilpotent Jacobson radical which is not right artinian.

3.4. Proposition. Let $R$ be a direct sum of finitely many local left artinian rings. Then:

(a) $\operatorname{dim}[E(R / P) / R / P] \geqq 1$ for every prime ideal $P$ which is a large left ideal.

(b) $\operatorname{dim}[E(R / J) / R / J]$ is not smaller than the number of prime ideals which are large left ideals.

Proof. (a) Let $P$ be a prime ideal with $\operatorname{dim}[E(R / P) / R / P]=0$. Then $R / P=E(R / P)$ and $R / P$ is injective. Since $\operatorname{ch}\left({ }_{R} R\right)=\operatorname{supp}\left({ }_{R} R\right)=\{P \mid P$ any prime ideal $\}$ by [6, Satz 4.2] and since $R$ is a Matlis ring, $R / P$ can be imbedded in $R$. Thus $R \cong_{R} R / P \oplus X$ for some left ideal $X$. Since $R / P$ is a simple module, 
$X$ is a maximal left ideal and therefore a prime ideal by [7, Theorem 3.6.] Since $R / X \cong{ }_{R} R / P$, it follows that $X=(R / X)_{l}=(R / P)_{l}=P$, so that $P$ is not large in $R$. (b) follows immediately from (a) and from the fact that $E(R / J) / R / J$ $\cong \bigoplus_{i=1}^{n}\left[E\left(R / P_{i}\right) / R / P_{i}\right]$, where the $P_{i}$ denote the finitely many prime ideals of $R$.

If $\operatorname{dim}[E(R / J) / R / J]$ is equal to the lower bound given in 3.4, i. e. if it is equal to the number of prime ideals which are large left ideals, then much information can be obtained about the structure of the injective indecomposable left $R$-modules.

3.5. THEOREM. The following properties of the ring $R$ are equivalent:

(1) $\left\{\begin{array}{l}\text { (a) } R \text { is a Matlis ring. } \\ \text { (b) } R \text { has the Artin-Rees-property for left ideals. } \\ \text { (c) Injective indecomposable left } R \text {-modules are cyclic and their } \\ \text { submodules are linearly ordered. }\end{array}\right.$

(2) $\left\{\begin{array}{l}\text { (a) } R \text { is a direct sum of finitely many local left artinian rings. } \\ \text { (b) } \operatorname{dim}[E(R / J) / R / J] \text { is equal to the number of prime ideals } \\ \text { which are large left ideals. }\end{array}\right.$

ProOf. (1) $\rightarrow$ (2): By 3.2 it is clear that (1) implies (2a). Let $P_{1}, \cdots, P_{n}$ denote the prime ideals of $R$. Since $R / J \cong_{R} \bigoplus_{i=1}^{n} R / P_{i}$ and $E(R / P) \cong_{R} \bigoplus_{i=1}^{n} E\left(R / P_{i}\right)$ by 2.1 , it follows that $\operatorname{dim}[E(R / J) / R / J]=\sum_{i=1}^{n} \operatorname{dim}\left[E\left(R / P_{i}\right) / R / P_{i}\right]$. Since the submodules of $E\left(R / P_{i}\right)$ are linearly ordered, either $E\left(R / P_{i}\right)$ is simple or there exists a unique minimal submodule of $E\left(R / P_{i}\right)$ which contains $R / P_{i}$ properly. Therefore, it follows that $\operatorname{dim}\left[E\left(R / P_{i}\right) / R / P_{i}\right]$ is either 1 or 0 . In order to prove $(2 \mathrm{~b})$ it thus suffices to show that $\operatorname{dim}\left[E\left(R / P_{i}\right) / R / P_{i}\right]=1$ iff $P_{i}$ is a large left ideal. By Proposition 3.4 it is clear that $\operatorname{dim}[E(R / P) / R / P]=1$ if the prime ideal $P$ is a large left ideal. Assume $P$ is not large. Since $R$ is a Matlis ring, every nonzero submodule of $E(R / P)$ contains an element $\neq 0$ whose annihilator is $P(\operatorname{cf}[7$, Theorem 2.7]). Thus $Z(E(R / P))=0$, whence $C(E(R / P))$ $=E(R / P)$ by $[9$, Propriété 3. 4]. Since $C(E(R / P))=S(E(R / P))=R / P$ by [7, Theorem 4.2] this implies $\operatorname{dim}[E(R / P) / R / P]=0$.

$(2) \rightarrow(1)$ : Assume the first $k$ among the prime ideals $P_{1}, \cdots, P_{n}$ are large left ideals. Since $\operatorname{dim}\left(E\left(R / P_{i}\right) / R / P_{i}\right) \geqq 1$ for all $i=1, \cdots, k$ by 3.4 and since $\operatorname{dim}[E(R / J) / R / J]=k$, it follows from $E(R / J) / R / J \cong \bigoplus_{i=1}^{n}\left[E\left(R / P_{i}\right) / R / P_{i}\right]$ that 
$P_{i}$ is a large left ideal if and only if $\operatorname{dim}\left[E\left(R / P_{i}\right) / R / P_{i}\right]=1$. Let $E=E(R / P)$ be an injective indecomposable left $R$-module. If $P$ is not large, $\operatorname{dim}[E(R / P) / R / P]$ $=0$, whence $E(R / P)$ is simple. If $P$ is large, let $S_{1}(E)=S(E)$ and define $S_{i+1}(E) / S_{i}(E)=S\left(E / S_{i}(E)\right)$ for $i \geqq 1$. Then $\operatorname{dim}\left[E / S_{1}(E)\right]=\operatorname{dim}[E(R / P) / R / P]=1$. Assume $\operatorname{dim}\left[E / S_{i}(E)\right] \leqq 1$ for $i \geqq 1$. Since $E / S_{i+1}(E) \cong E / S_{i}(E) / S_{i+1}(E) / S_{i}(E)$ $\subseteq E\left(E / S_{i}(E)\right) / S_{i+1}(E) / S_{i}(E) \cong E(R / P) / R / P$, we conclude that $\operatorname{dim}\left(E / S_{i+1}(E)\right) \leqq 1$. Therefore, all the factors of the Loewy-series $0 \subset S_{1} \subset \cdots S_{m}=E$ are simple. If $A \neq 0$ is a proper submodule of $E$ and $S_{i}$ the largest member of the Loewyseries contained in $A$, then $A=S_{i}$ since otherwise $S_{i+1} / S_{i} \subseteq A / S_{i}$, yielding $S_{i+1} \subseteq A$. Therefore the members of the Loewy-series are the only submodules of $E$. Clearly $E$ is cyclic, every element $e \notin S_{m-1}$ generates $E$.

3. 6. REMARK. From the proof of 3.5 it is clear that if $\operatorname{dim}[E(R / J) / R / J]$ $=k$ equals the number of prime ideals which are large left ideals, then this number is also equal to the number of non-isomorphic non-simple injective indecomposable left $R$-modules. Furthermore, if under the above circumstances $n$ denotes the total number of prime ideals, it is easy to see that then $R$ is a direct sum of $n$ - $k$ division rings and $k$ local left artinian rings which are not division rings. The special case $k=0$ may be of some interest, and we add another module theoretic condition necessary and sufficient for a direct sum of finitely many local left artinian rings to be a direct sum of division rings.

3. 7. COROLLARY. The following properties of the ring $R$ are equivalent:

(1) $\left\{\begin{array}{l}\text { (a) } R \text { is a reduced left artinian ring. } \\ \text { (b) The left } R \text {-module } R / J \text { is injective. }\end{array}\right.$

(2) $R$ is a direct sum of finitely many division rings.

(3) $\left\{\begin{array}{l}\text { (a) } R \text { is a direct sum of finitely many local left artinian rings. } \\ \text { (b) Extensions of small modules by small modules are small } \\ \text { modules. }\end{array}\right.$

PROOF. (1) $\rightarrow(2):$ By Proposition $2.1, R / J \cong_{R} \bigoplus_{i=1}^{n} R / P_{i}$, where $P_{1}, \cdots, P_{n}$ denote the prime ideals of $R$. Thus each $R / P_{i}$ is an injective $R$-module. Since prime ideals of $R$ are maximal left ideals and $R$ is a Matlis ring, it follows that all injective indecomposable left $R$-modules are simple. Since $R$ is left noetherian, this implies that all left $R$-modules are semi-simple (cf [11, Theorem $2.5]$ ), so that $R$ is a semi-simple Matlis ring with minimum condition whence (2) follows from Corollary 1.6. (2) $\rightarrow(3)$ : Trivial, since every module over a 
semi-simple artinian ring is semi-simple. Therefore small modules $\neq 0$ do not exist and (3b) is fulfilled vacously.

$(3) \rightarrow(1)$ : It is clear that (1a) holds. Let $E$ be an injective indecomposable left $R$-module. Since $R$ is left artinian, $S(E) \neq 0$. Since $S(E)$ is the unique minimal submodule of $E$, either $S(E)=E$ or $S(E)$ is small in $E$. We shall prove that the second case cannot occur. Let again be $S_{1}(E)=S(E)$ and define $S_{i+1}(E) / S_{i}(E)=S\left(E / S_{i}(E)\right)$ for $i \geqq 1$. Assume $S_{i}(E)$ is a small submodule of $E$. Since $\operatorname{ch}\left(E / S_{i}(E)\right)=\operatorname{supp}\left(E / S_{i}(E)\right) \subseteq \operatorname{supp}(E)=\operatorname{ch}(E)$ by [6, Satz 4.2] and since $R$ is a Matlis ring, it follows from [6, Folgerung 2.8] that $E\left(E / S_{i}(E)\right)$ is a direct sum of modules isomorphic to $E$. Since $S(E)=S_{1}(E)$ is small in $E$ by assumption, this implies that $S_{i+1}(E) / S_{i}(E)=S\left(E / S_{i}(E)\right)=S\left(E\left(E / S_{i}(E)\right)\right)$ is small in $E\left(E / S_{i}(E)\right)$ by Lemma 1.7. Since $S_{i}(E)$ is small by hypothesis, it follows from (3b) that $S_{i+1}(E)$ is a small module and hence a small submodule of $\mathrm{E}$ by [8, Theorem 1]. Thus every $S_{i}(E)$ is small in $E$. Since $J(R)$ is nilpotent, $E=S_{n}(E)$ for some $n$, which leads to a contradiction since $E$ is not small in $E$. Thus $E$ is simple, and hence $R / P$ injective for every prime ideal $P$. By Proposition 2.1 this implies (1b).

\section{The case of a ring which is commutative modulo the square of its Jacobson radical.}

4. 1. THEOREM. Let $R$ be a ring such that $R / J^{2}$ is commutative. Then the following properties of $R$ are equivalent:

(1) $R$ is left artinian.

(2) $R$ is a direct sum of finitely many local left artinian rings.

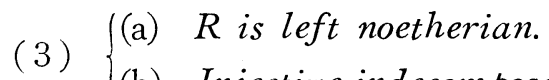

(b) Injective indecomposable left R-modules are finitely generated.

Proof. (1) $\rightarrow(2)$ : Since $R$ is left artinian, its Jacobson radical is equal to the lower Baer nil radical. Thus $J$, and hence in particular $J^{2}$ is contained in every prime ideal. Since $R / J^{2}$ is commutative, this implies that prime ideals of $R$ are maximal left ideals. Thus $R$ is a Matlis ring by [6, Hilfssatz 4.1] and [7, Theorem 2.7] and hence a reduced left artinian ring by Theorem 1.5. In order to establish (2) it suffices to show that $S(M)=C(M)$ for every left $R$-module $M$ (cf. [7, Theorem 4.2]). By [6, Folgerung 3.10] this is true if and only if $S(E)=C(E)$ for all injective indecomposable modules $E$. Let $E$ be such a module and let $E=E_{R}(R / P)$ for a prime ideal $P$. Since $S(E)=P_{r}(E)$ and $P_{r}(E) \subseteq C(E)$ by [6, Lemma 3.5], it suffices to show that $C(E) \subseteq P_{r}(E)$. Since $P_{r}(E)$ is a large submodule of $E$, it is sufficient to show that $E\left(E / P_{r}(E)\right)$ is a 
direct sum of injective indecomposable modules which are all isomorphic to $E$ (cf. [6, Satz 3.1]. Since $R$ is a Matlis ring this will follow if we can show that $\operatorname{ch}\left(E / P_{r}(E)\right)=\{P\}$ (cf.[6, Folgerung 2.8]). Let therefore $Q \in \operatorname{ch}\left(E / P_{r}(E)\right.$ ). Then there exists an element $0 \neq \bar{x}=x+P_{r}(E) \in E / P_{r}(E)$ with $Q=\bar{x}_{l}$. Thus $Q x \subseteq P_{r}(E)$, whence $P Q x=0$. Therefore, $J^{2} \subseteq x_{l}$, and since $R / J^{2}$ is commutative, it follows that $Q P x=0$, whence $Q \subseteq(P x)_{l}$. Since $x \notin P_{r}(E), P x \neq 0$, so there exists a submodule $0 \neq N \subseteq P x$ such that $N_{l}=P$. Thus $Q \subseteq(P x)_{l} \subseteq N_{l}=P$, whence $Q=P$, since prime ideals of $R$ are maximal.

$(2) \rightarrow(3):$ In view of Theorem 2.2 it suffices to show that $\operatorname{dim}[E(R / J) / R / J]$ is finite. Since $E(R / J) / R / J \cong_{R} \bigoplus_{i=1}^{n}\left[E\left(R / P_{i}\right) / R / P_{i}\right]$ by Proposition 2.1, where $P_{1}, \cdots, P_{n}$ denote the finitely many prime ideals of $R$, it will suffice to show that $\operatorname{dim}[E(R / P) / R / P]$ is finite for every prime ideal $P$ of $R$. This is true if and only if the socle of $E(R / P) / R / P$ is the direct sum of finitely many simple modules. Since $\operatorname{supp}(M)=\operatorname{ch}(M)$ for every left $R$-module $M$ by[6, Satz 4.2], we get

$$
\operatorname{ch}(E(R / P) / R / P)=\operatorname{supp}(E(R / P) / R / P) \subseteq \operatorname{supp}(E(R / P))=\operatorname{ch}(E(R / P))=\{P\} .
$$

Since $P$ is a maximal left ideal, this implies that

$$
S(E(R / P) / R / P)=P_{r}^{2}(E(R / P)) / P_{r}(E(R / P))
$$

For simplicity let $E=E(R / P)$. By [12, Lemma 1$], \quad P_{r}^{2}(E) / P_{r}(E)$ $\cong_{R} \operatorname{Hom}_{R}\left(P / P^{2}, E\right)$, where $\operatorname{Hom}_{R}\left(P / P^{2}, E\right)$ is a left $R$-module by virtue of the right operations of $R$ on $P / P^{2}$, viz $\bar{p}(r \phi)=(\bar{p} r) \phi$ for $r \in R, \bar{p} \in P / P^{2}, \phi$ $\in \operatorname{Hom}_{R}\left(P / P^{2}, E\right)$. Since $P / P^{2}$ is a left $R / P$-module and since $R / P$ is a division ring, $P / P^{2}$ is a semi-simple $R / P$-module and hence also a semi-simple left $R$-module. Since images of semi-simple modules are semi-simple and since $S(E) \cong R / P$, it follows that

$$
\operatorname{Hom}_{R}\left(P / P^{2}, E\right)=\operatorname{Hom}_{R}\left(P / P^{2}, S(E)\right) \cong{ }_{R} \operatorname{Hom}_{R}\left(P / P^{2}, R / P\right)
$$

Since $R$ is left noetherian, $P / P^{2}$ is a direct sum of finitely many simple modules $X_{i}, i=1, \cdots, \mathrm{m}$. Since $\left(X_{i}\right)_{l} \supseteq\left(P / P^{2}\right)_{l}=P$ for every $i$ and since $P$ is a maximal left ideal, $X_{i} \cong R / P$ for every $i=1, \cdots, n$. Let now $\phi$ be an $R$-isomorphism of the direct sum of $m$ copies of the left $R$-module $R / P$ onto $P / P^{2}$. Then we define a mapping

$$
\psi: \operatorname{Hom}_{R}\left(P / P^{2}, R / P\right) \rightarrow \operatorname{Hom}_{R}\left(\bigoplus_{i=1}^{m} R / P, R / P\right)
$$


by $f \dot{\psi}=\phi f$ for every $f \in \operatorname{Hom}_{R}\left(P / P^{2}, R / P\right)$. Clearly $f$ is well-defined, additive, one-to-one and onto. To show that $\psi$ is an $R$-homomorphism, let $a \in \bigoplus_{i=1}^{m} R / P$ and $r \in R$. Then

$$
a[(r f) \psi]=a[\varphi(r f)]=(a \phi)(r f)=((a \varphi) r) f
$$

where the last equation results from the definition of the left operation of $R$ on $\operatorname{Hom}_{R}\left(P / P^{2}, R / P\right)$. Since $R / J^{2}$ is commutative, $R / P^{2}$ is commutative, whence $s r-r s$ $\in P^{2}$ for all elements $r, s \in R$. Since $a \varphi \in P / P^{2}$ and since $\phi$ is an $R$-homomorphism, we get $((a \varphi) r) f=(r(a \varphi)) f=((r a) \varphi) f$. Since $a \in \bigoplus_{i=1}^{m} R / P$ and since $R / P$ is commutative, $r a=a r$, whence $((r a) \phi) f=((a r) \phi) f=(a r)(\phi f)=(a r)(f \psi)$ $=a(r(f \psi))$, where the last equation follows from the definition of the left operation of $R$ on $\operatorname{Hom}_{R}\left(\bigoplus_{i=1}^{m} R / P, R / P\right)$. Putting these equations together it follows that $\psi$ is an $R$-isomorphism, whence $\operatorname{Hom}_{R}\left(P / P^{2}, R / P\right) \cong_{R} \operatorname{Hom}_{R}\left(\bigoplus_{i=1}^{m} R / P, R / P\right)$. Let now $f=\sum_{i=1}^{m} f_{i} \in \bigoplus_{i=1}^{m} \operatorname{Hom}_{R}(R / P, R / P)$ and define $\eta: \bigoplus_{i=1}^{m} \operatorname{Hom}_{R}(R / P, R P)$ $\rightarrow \operatorname{Hom}_{R}\left(\bigoplus_{i=1}^{m} R / P, R / P\right)$ by $a(f \eta)=\sum_{i=1}^{m} a \pi_{i} f_{i}$ for all $a \in \bigoplus_{i=1}^{m} R / P$, where $\pi_{i}$ denotes the canonical projection of $\bigoplus_{i=1}^{m} R / P$ onto its $i$-th summand. It is wellknown that $\eta$ is a group isomorphism of $\bigoplus_{i=1}^{m} \operatorname{Hom}_{R}(R / P, R / P)$ onto $\operatorname{Hom}_{R}\left(\bigoplus_{i=1}^{m} R / P, R / P\right)$. In order to establish the fact that $\eta$ is also an $R$. homomorphism, let $r \in R$ and $a \in \bigoplus_{i=1}^{m} R / P$. Then $a((r f) \eta)=\sum_{i=1}^{m}\left(a \pi_{i}\right)\left(r f_{i}\right)$ $=\sum_{i=1}^{m}\left(\left(a \pi_{i}\right) r\right) f_{i}$ by the definition of the left operation of $R$ on $\operatorname{Hom}_{R}(R / P, R / P)$. Since $a \pi_{i} \in R / P$ and since $R / P$ is a commutative ring, $\left(a \pi_{i}\right) r=r\left(a \pi_{i}\right)$. Since $\pi_{i}$ is an $R$-homomorphism we thus get

$$
\sum_{i=1}^{m}\left(\left(a \pi_{i}\right) r\right) f_{i}=\sum_{i=1}^{m}\left(r\left(a \pi_{i}\right)\right) f_{i}=\sum_{i=1}^{m}\left((r a) \pi_{i}\right) f_{i} .
$$

Since $a \in \bigoplus_{i=1}^{m} R / P, \quad a$ commutes with $r$, whence $\sum_{i=1}^{m}(r a) \pi_{i} f_{i}=\sum_{i=1}^{m}(a r) \pi_{i} f_{i}$ $=(a r)(f \eta)=a(r(f \eta))$, where the last equation follows again from the definition of the left operation of $R$ on $\operatorname{Hom}_{R}\left(\bigoplus_{i=1}^{m} R / P, R / P\right)$. Thus $\eta$ is an $R$-isomorphism. 
Putting all these isomorphisms together, it follows that

$$
P_{r}^{2}(E) / P_{r}(E) \cong_{R} \bigoplus_{i=1}^{m} \operatorname{Hom}(R / P, R / P)
$$

Since images of simple modules are simple, it follows that $\operatorname{Hom}_{R}(R / P, R / P)$ $=\operatorname{Hom}_{R}(R / P, E(R / P))$, whence $\operatorname{Hom}_{R}(R / P, R / P) \cong P_{r}(E) / R_{r}(E)=P_{r}(E) / 0=S(E)$ by [12, Lemma 1]. Therefore $P_{r}^{2}(E) / P_{r}(E)$ is $R$-isomorphic to a direct sum of $m$ copies of $S(E)$, which implies that $E(R / P) / R / P$ has finite Goldie-dimension.

$(3) \rightarrow(1)$ : This follows from [4, Corollary 2.3].

4. 2. REMARK. Theorem 4.1 is false, if we only require that $R / J$ is commutative. The ring of Example 2.3 is commutative modulo its Jacobson radical, but not commutative modulo the square of its Jacobson radical since this is equal to 0 . On the other hand, the fact $R / J^{2}$ is commutative was only needed to prove $(1) \rightarrow(2)$ in 4.1 . In the proof from (2) to (3) we only used the weaker condition that $R$ is commutative modulo the intersection of the squares of its prime ideals. Therefore the question arises, whether this weaker condition is also sufficient to get from (1) to (2).

\section{REFERENCES}

[ 1 ] G. AzumayA, Corrections and supplementaries to my paper concerning Krull-RemakSchmidt's theorem, Nagoya Math. J., 1(1950), 117-124.

[2] H. BAss, Finitistic dimension and a homological generalization of semi-primary rings, Trans. Amer. Math. Soc., 95(1960), 466-488.

[ 3 ] C. FAITH AND Y. UTUMI, Quasi-injective modules and their endomorphism rings, Arch. Math., 15(1964), 166-174.

[4] C. FAITH AND E. A. WALKER, Direct-sum representations of injective modules, J. Algebra, $5(1967), 203-221$.

[5] A. W. GoldIE, Semi-prime rings with maximum condition, Proc. London Math. Soc., (3) 10(1960), 201-220.

[6] G. KRAUSE, Moduln über linksnoetherschen Ringen, ihr Herz und Sockel, ihre Charakteristik und ihr Träger, Journ. f. d. Reine u. Angew. Math., 235(1969), 133-149.

[7] G. KRAUSE, Admissible modules and a characterization of reduced left artinian rings, Pacific J. Math., 33(1970), 291-309.

[ 8 ] W. W. Leonard, Small modules, Proc. Amer. Math. Soc., 17(1966), 527-531.

[9] L. Lesieur AND R.CRoisot, Coeur d'un module, J. Math. Pures Appl., 42(1963), 367-407.

[10] L. LESIEUR AND R. CROISOT, Algèbre noethérienne non commutative, Mémor. Sci. Math., 154(1963).

[11] E. MATLIS, Injective modules over noetherian rings, Pacific J. Math., 8(1958), 511-528.

[12] A. Rosenberg AND D.Zelinsky, Finiteness of the injective hull, Math. Z., 70(1959), $372-380$.

DEPARTMENT OF MATHEMATICS

WASHINGTON STATE UNIVERSITY

PUllmaN, WASH., U.S.A. 